\title{
RELACION ENTRE EL ESTRÉS OXIDATIVO DIABÉTICO Y LA ENFERMEDAD PERIODONTAL. ESTUDIO EXPERIMENTAL
}

\author{
RELATION BETWEEN DIABETES OXIDATIVE STRESS \\ AND PERIODONTAL DISEASE
}

Orlando Catanzaro ${ }^{1,2,3}$, Jorgelina Aira Capponi³ ${ }^{3}$ Irene Di Martino' ${ }^{1}$, Emilio Labal ${ }^{3}$

\begin{abstract}
RESUMEN
Introducción: la diabetes mellitus comprende un grupo de desórdenes caracterizados por la alterada tolerancia a la glucosa y el deterioro del metabolismo de lípidos y carbohidratos. La enfermedad diabética se asocia con numerosas complicaciones directamente resultantes de la hiperglucemia. Dentro de las complicaciones, una de las más importantes es la enfermedad periodontal (EP) por los procesos de estrés oxidativo causados por la patología diabética. El objetivo de este trabajo es describir el efecto de la patología diabética sobre los factores antioxidantes y oxidantes que afectan a la enfermedad periodontal. Materiales y métodos: ratas Wistar machos fueron inyectadas con streptozotocin (STZ,65mg/kg). Se obtuvieron muestras de encía y sangre para determinaciones bioquímicas. Al final de la cuarta semana, en las muestras se determinaron las glucemias, peroxidación lipídica medida por el malondialdehído (MDA), glutatión reducido (GSH) y óxido nítrico (ON).

Resultados: la diabetes produjo cambios significativos en las glucemias como así también en el estrés oxidativo en encía y plasma, con aumento de la peroxidación lipídica (MDA) y ON. En cuanto a la actividad antioxidante de GSH, se observó una significativa reducción en encía y plasma comparada con los controles. Conclusión: la diabetes afecta todos los parámetros oxidantes y antioxidantes del plasma y la encía, disminuyendo la capacidad de defensas y causando daños en los tejidos periodontales.
\end{abstract}

Palabras clave: diabetes, enfermedad periodontal, estrés oxidativo.

Revista de la Sociedad Argentina de Diabetes 2014; Vol. 48 (70-74)

1 Escuela de Odontología, Universidad Argentina John F. Kennedy (UAJFK)

2 Escuela de Medicina y Odontología, Universidad del Salvador (USAL), AOA

${ }^{3}$ Laboratorio de Diabetes Experimental - Departamento de Biología, UAJFK

Contacto del autor: Orlando Catanzaro, Director del Laboratorio de Diabetes Experimental, UAJFK. Prof. Emérito de Medicina, USAL

E-mail: ocatanzaro@hotmail.com.ar/Tel.: 15-5632-7079 Correspondencia: Sarmiento 4564 (C1197ABA), CABA Este trabajo fue realizado con subsidios de la Escuela de Odontología y Bioquímica de la UAJFK y de la Escuela de Medicina y Odontología de la USAL ( A 233/12-15 y AOA 117/13) Fecha de trabajo recibido: 4/2/14

Fecha de trabajo aceptado: 24/6/14

\begin{abstract}
Introduction: diabetic mellitus comprises a heterogeneous disorder characterized by hyperglycemia and impaired lipid and carbohydrate metabolism. The diabetic disease is associated with a number of complications, resulting directly from hyperglycemia. Among others, periodontal disease is a very important complication of diabetes. Oxidative stress plays an important role in the pathogenesis of periodontal disease.

Material and methods: Wistar male rats were injected with STZ $(65 \mathrm{mg} / \mathrm{kg})$. At the end of week 4, gingival blood were obtained for biochemical determinations. Oxidative changes were determined by glutathione activity (GSH), lipid peroxidase (MDA), nitric oxide (NO) and glycaemia.

Results: STZ treatment produced significant changes in glycaemia, plasma and gingival tissue MDA and NO compared to control. The antioxidant activity GSH was significantly reduced in plasma and gingival tissue with respect to controls.

Conclusion: diabetes affects all oxidant and antioxidant parameters in plasma and gingival tissue decreasing the defense capacity and causing damage to periodontal tissue.
\end{abstract}

Key words: diabetes, periodontal disease, oxidative stress.

Revista de la Sociedad Argentina de Diabetes 2014; Vol. 48 (70-74)

\section{INTRODUCCIÓN}

La diabetes mellitus es un desorden metabólico que ocurre en diversas formas y se caracteriza por una anormal regulación del metabolismo de la glucosa. En los pacientes diabéticos la reparación del tejido es una secuencia de eventos que involucran inflamación, angiogénesis, remodelación y reparación completa del tejido. Algunos mecanismos moleculares han sido propuestos para tener en consideración en estos efectos patológicos. En particular dos mecanismos tienen importancia en el progreso de la enfermedad periodontal (EP) diabética: la acumulación de productos finales de la glucosilación avanzada en las células expuestas a hiperglucemia crónica, y el daño provocado por el estrés oxidativo resultante de la superproducción de especies reactivas de oxigeno (ERO) 1,2,3,4. La glucosilación y gliooxidación de proteínas a pro- 
ductos finales de la glucosilación avanzada (AGEs) tiene estricta dependencia con los procesos oxidativos ${ }^{5}$ que ocurren en la diabetes ${ }^{6}$ como uno de los factores de mayor riesgo de periodontitis. Las reacciones de oxidación y reducción se consideran comúnmente bajo el término de redox. ¿Cómo participa esta reacción en la EP diabética? El término estrés oxidativo describe condiciones que involucran el nivel de las llamadas especies reactivas de oxígeno (ERO); ; a su vez este concepto se aplica para explicar la relación entre la enfermedad periodontal y las condiciones sistémicas diferentes tales como la diabetes ${ }^{7}$, el síndrome metabólico ${ }^{8}$, la disfunción mitocondrial ${ }^{9}$ y la artritis reumatoidea ${ }^{10}$ donde ocurren cambios sistémicos.

Dentro de la bolsa periodontal, un bajo potencial redox puede ser observado como elemento principal de los anaerobios subgingivales ${ }^{11}$, en tanto que dentro de las células y tejidos, un bajo potencial redox es considerado protectivo contra el estrés oxidativo. Cuando este balance se rompe, aumenta la producción de radicales libres y se reduce la actividad de las defensas antioxidantes ${ }^{12}$. Existen importantes evidencias de una significativa relación entre la hiperglucemia, el estrés oxidativo y las lesiones gingivales y óseas en la enfermedad periodontal ${ }^{13}$.

El presente estudio experimental analiza la patología diabética y la importancia del estrés oxidativo estimando los niveles de especies reactivas de oxígeno en la encía de ratas diabéticas durante el progreso de la inflamación periodontal, comparado con controles no diabéticos.

\section{MATERIALES Y MÉTODOS}

- Diseño: el diseño fue experimental, retrospectivo y comparativo.

- Consideraciones bioéticas: el protocolo de estudio fue aprobado por el Comité de Bioética de la Universidad Argentina John F. Kennedy (149/12).

- Criterios de selección: los animales fueron seleccionados de cepa Wistar machos del Instituto Malbrán (Buenos Aires, Argentina), con entre 200 y $220 \mathrm{~g}$ de peso corporal e inyectados con una dosis i.p. de STZ de $65 \mathrm{mg} / \mathrm{Kg}$ de peso corporal, (Streptozotocin, SIGMA-Aldrich, Estados Unidos), disuelta en 0,05 M de citrato buffer $\mathrm{pH}$ 7,5 ó vehículo en controles adecuados.

- Criterios de exclusión: se excluyeron animales con glucemias superiores a 16+/-1,3 mmol/L.

Los animales fueron divididos en dos grupos: 1) Controles ( $n=9)$; 2) Diabéticos ( $n=9)$ manteni- dos con control de temperatura $\left(23+/-2^{\circ} \mathrm{C}\right)$, y alimentación y agua ad limitum durante las cuatro semanas del experimento.

- Maniobras: previo a la cirugía, se obtuvo sangre de la arteria de la cola para determinar las glucemias de ratas diabéticas y de controles por medio de un analizador automático (Contour TS, Bayer Diagnostic, Argentina). A las cuatro semanas, los animales 1) Controles y 2) Diabéticos fueron anestesiados con thiopental sódico $(40 \mathrm{mg} /$ $\mathrm{kg})$. Se obtuvieron muestras sangre en heparina por punción cardíaca y las encías del sector incisal y molar fueron extraídas con micro bisturí.

Las muestras de sangre extraídas con heparina se centrifugaron y se extrajo el plasma para determinar los niveles de glucosa, glutatión reducido (GSH), lípido peroxidasa (MDA) y óxido nítrico (ON).

Las muestras de encías fueron homogeneizadas en $10 \mathrm{mM}$ Tris- $\mathrm{HCl}$, pH 7,4, centrifugadas y obtenido el sobrenadante para la determinación de proteínas $^{14}, \mathrm{GSH}^{15}, \mathrm{MDA}^{16}$ y óxido nítrico $(\mathrm{ON})^{17}$. Todas las muestras se determinaron por triplicado. El grupo de animales controles fue tratado quirúrgicamente en la misma forma que los diabéticos.

- Análisis de datos: todas las muestras fueron analizadas por ANOVA por test de Tukey post hoc para múltiples comparaciones y Student's test. Los resultados fueron expresados como la media \pm ES. Las diferencias fueron consideradas significativas con un $\mathrm{P}<0,05$.

\section{RESULTADOS}

Las ratas diabéticas exhibieron un significativo aumento de la concentración de glucosa plasmática comparado con controles: C: 5,6+/- 1,2; D: 16,1+/- 1,3 mmol/L; $P<0,05$ (Tabla 1). Los resultados de la peroxidación lipídica, específicamente el malondialdehído (MDA), en los animales diabéticos mostraron aumento significativo con respecto a los controles: C: 12,6+/-.5; D: 24,4+/-1,5 nmol/ $\mathrm{mgP}(\mathrm{P}<0,05)$ y una disminución de GSH en el plasma de ratas diabéticas comparado con el control (C: 25,9+/-2,1; D: 14+/-1,2 nmol/100mgP, $P<0,05)$; en tanto que la actividad de ON fue $\mathrm{C}$ : $6+/-1,2$, $\mathrm{D}: 11,3+/-1,3 \mathrm{nmol} / \mathrm{mgP}, \mathrm{P}<0,05$ (Tabla 1).

Por otra parte, la actividad de peroxidasa lipídica (MDA) en encía de ratas diabéticas fue significativamente aumentada respecto de controles. 1) Controles 8,52+/-.6; 2) Diabéticas: $13,5+/-1,2$ $\mathrm{nmol} / \mathrm{mg} \mathrm{P}_{\text {, }}(\mathrm{P}<0,05)$ (Figura 1); en tanto que el glutatión reducido (GSH) disminuyó significativa- 
mente respecto de Controles: C: 9,9+/7, D: 5,5+/1, nmol/100mg $P$, $(P<0,05)$ (Figura 2). El estrés oxidativo también mostró diferencias en la concentración de óxido nítrico de controles y diabéticos: ON: C: 2,69+/-.4; D: 6,83+/-1,1 nmol/mgP, $(\mathrm{P}<0,05)$ (Figura 3).

\begin{tabular}{|l|r|r|r|r|}
\hline & Glucemias & \multicolumn{1}{c|}{ MDA } & \multicolumn{1}{c|}{ GSH } & \multicolumn{1}{c|}{ ON } \\
\hline & $\mathrm{mM} / \mathrm{L}$ & $\mathrm{nmol} / \mathrm{mgP}$ & $\mathrm{nmol} / 100 \mathrm{mgP}$ & $\mathrm{nmol} / \mathrm{mgP}$ \\
\hline Control & $5,6+/-1,2$ & $12,6+/-.5$ & $25,9+/-2,1$ & $6+/-1,2$ \\
\hline Diabéticas & $16,1+/-1,3^{*}$ & $24,4+/-1,5^{*}$ & $14+/-1,2 *$ & $11,3+/-1,3 *$ \\
\hline
\end{tabular}

Tabla 1: Concentración de glucosa plasmática, lípido peroxidasa (MDA), glutatión reducido (GSH) y óxido nítrico (ON) en ratas Controles y Diabéticas de 30 días. Cada muestra representa $+/-\operatorname{SEM}(n=9)$. ${ }^{*} \mathrm{P}<0,05$.

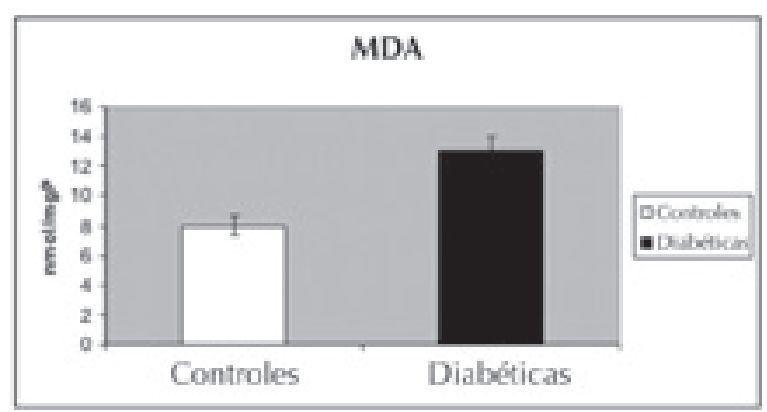

Figura 1: Actividad de lípido peroxidasa (MDA) en encía de ratas Controles y Diabéticas (30 días). Los valores son la media +/- SEM. ( $n=9$ por cada grupo). * $\mathrm{P}<0,05$ Diabéticas vs. Controles.

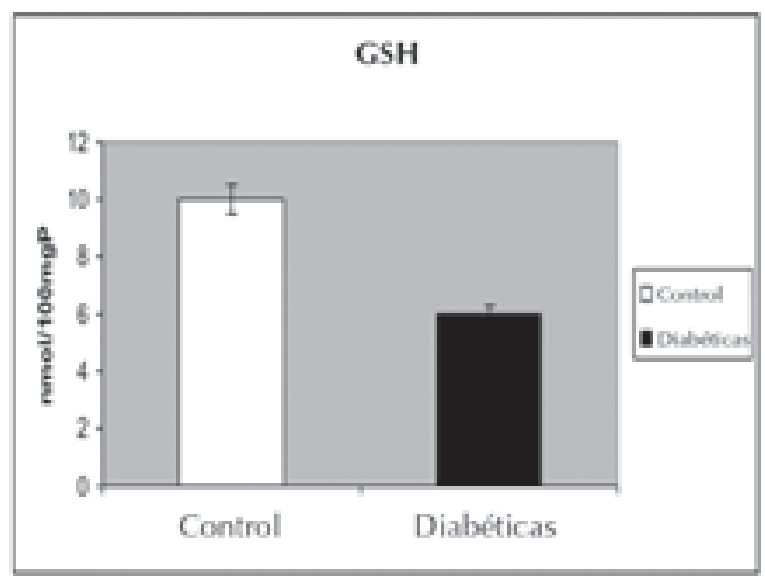

Figura 2: Actividad de glutatión reducido (GSH) en encía de ratas Controles y Diabéticas (30 días) ( $n=9$ ratas por grupo). Los valores son las medias +/-SEM. ${ }^{*} \mathrm{P}<0,05$ Controles vs. Diabéticas.

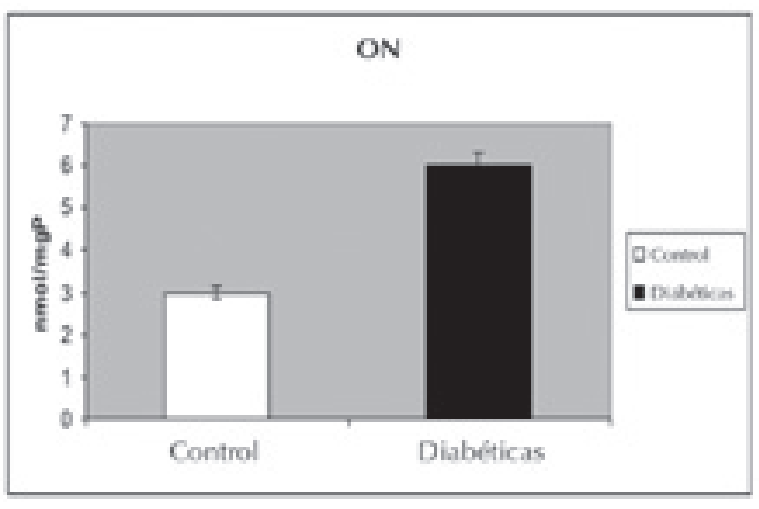

Figura 3: Actividad de óxido nítrico en encía de ratas Controles y Diabéticas (30 días) ( $\mathrm{n}=9$ por grupo). Los valores representan la media +/-SEM. ${ }^{*} \mathrm{P}<0,05$ Controles vs. Diabéticas.

\section{DISCUSIÓN}

Los resultados del presente estudio indican que el estrés oxidativo produce cambios significativos en plasma y encía de ratas durante la evolución de la diabetes. La diabetes mellitus (desorden metabólico caracterizado por hiperglucemia) y la periodontitis (desorden inflamatorio que afecta las estructuras de soporte dentario) tienen en común condiciones crónicas de estrés oxidativo ${ }^{18}$.

El estrés oxidativo juega un rol importante en la patogénesis y las complicaciones de la diabetes mostrando que la hiperglucemia resulta en una superproducción de radicales libres de oxígeno los cuales contribuyen a las complicaciones de la diabetes ${ }^{19}$ como, por ejemplo, la enfermedad periodontal. La periodontitis crónica es una enfermedad inflamatoria con una respuesta caracterizada por exagerada inflamación, que involucra la liberación de enzimas proteolíticas y especies reactivas de oxígeno $(E R O)^{20}$. Los antioxidantes son definidos como aquellas sustancias, las cuales están presentes a bajas concentraciones comparadas con substratos oxidables ${ }^{21}$. En la fisiología normal hay un equilibrio dinámico entre especies reactivas oxidantes (ERO) y la capacidad de defensas antioxidantes, y cuando este equilibrio se desvía en sentido de $E R O$, ya sea por una reducción de las defensas antioxidantes o un aumento en la producción o actividad de ERO, indefectiblemente resulta en un proceso de estrés oxidativo.

Se han demostrado evidencias del rol crucial de ERO en la destrucción tisular de la enfermedad periodontal ${ }^{22}$. También se ha comprobado que indivi- 
duos que sufren periodontitis pueden tener un alto riesgo de desarrollar enfermedades crónicas inflamatorias, como enfermedades cardiovasculares y diabetes siendo ERO uno de los mecanismos de esta asociación. De acuerdo con Allen ${ }^{23}$, el estrés oxidativo se define como una modificación entre el balance de pro-oxidantes/antioxidantes a favor de los primeros, conduciendo a un daño potencial. El aumento de la producción de ERO durante la inflamación periodontal demostraría el rol de los antioxidantes en el estrés oxidativo ${ }^{24}$.

Tanto la diabetes como la EP son modulados por una respuesta inmune como, por ejemplo, aumento de citokinas pro-inflamatoiras e iniciación del daño tisular. Existen diversos mecanismos que podrían explicar la mayor incidencia de periodontitits diabética: por ejemplo, una gran susceptibilidad a infecciones como resultado de la función de neutrófilos en la diabetes, la formación de productos finales avanzados de la glucosilación, los cuales aumentan el estrés oxidativo en el tejido periodontal, y la unión de esos productos de la glucosilación a los receptores de la membrana celular lo cual estimula el aumento de citokinas inflamatorias (iniciado por bacterias) que causan lesiones irreversibles. Precisamente estos mecanismos podrían inducir en el estado diabético ${ }^{25,26}$ a una enfermedad periodontal con destrucción a través de osteoclastogenésis ${ }^{25}$. La interacción de estos mecanismos en el fluido de la bolsa gingival ${ }^{27}$ y en el periodonto con la preexistencia EP proporciona los parámetros necesarios de la destrucción periodontal de la diabetes y explica por qué los pacientes diabéticos tienen mucho más riesgo de EP.

La prevalencia de diabetes aumenta severamente después de los 45 años, siendo cerca del $25 \%$. Éste es un dato importante ya que la diabetes mellitus y la enfermedad periodontal son crónicas y comunes en la población, estando especialmente relacionadas en aquellos pacientes mayores de 65 años. Esta relación se potencia frente a la producción de radicales libres, ya que la diabetes mellitus es un factor de riesgo en las gingivitis y periodontitis por aumentar en severidad, progresión, como así también en la iniciación de la enfermedad periodontal. Es importante, entonces, tener en cuenta la función preventiva de antioxidantes por su efecto de remoción de superóxidos y peróxido de hidrógeno, o por remover iones metálicos divalentes y subsecuentemente la formación de radicales hidroxílicos.
Es fundamental reconocer que algunos antioxidantes tienen un efecto dual y algunas veces una acción triple. Por ejemplo, el ascorbato actúa como secuestrador antioxidante como así también preventivo antioxidante por su capacidad para reciclar $\alpha$-tocoferol (vitamina E) desde su forma oxidada ${ }^{28} y$ por su habilidad para unirse a iones metálicos. Lo mismo puede decirse de la vitamina $\mathrm{C}$ como antioxidante en enfermedades inflamatorias y la diabetes ${ }^{29,30}$. El fluido gingival es tres veces más elevado en vitamina $\mathrm{C}$ que la concentración en plasma ${ }^{31} \mathrm{y}$ ha sido demostrado para prevenir la colagenasa de los neutrófilos ${ }^{32}$.

En conclusión, lo observado en este estudio experimental demuestra que el estrés oxidativo producido por la diabetes incrementa significativamente el daño en la EP, y sugiere que la diabetes crónica induce mecanismos de destrucción que pueden ser irreversibles. Por otra parte, el efectivo tratamiento temprano -con un buen control de la hiperglucemia diabética y terapia antioxidante en la EP- puede mejorar el progreso de la enfermedad periodontal y consecuentemente el estado diabético y el estrés oxidativo. Estudios en realización por nuestro grupo de trabajo analizan el efecto de nuevos compuestos antioxidantes en la diabetes y la EP.

\section{REFERENCIAS}

1. Borges I Jr, Moreira EA, Filho DW, de Oliveira TB, da Silva MB, Frode TS. Proinflammatory and oxidative stress markers in patients with periodontal disease. Mediators Inflamm. 2007; 2007:45794.

2. Masi S, Salpea KD, Li K, Parkar M, Nibali I, Donos N, Patel K, Taddei S, Deanfield JE, D'Aiuto F, Humphries SE. Oxidative stress, chronic inflammation, and telomere length in patients with periodontal disease. Free radic. Biol. Med. 2011; 50:730-735.

3. Zizzi A,Tirabassi G, Aspriello SDS, Piemontese M, Rubini C, Lucarini G. Gingival advanced glycation end-products in diabetes mellitus- associated to immunohistochemical study. J. Periodontal Res. 2013; 48:293-301.

4. Duarte PM, Napimoga MH, Fagnani EC, Santos VR, Bastos MF, Ribeiro FV, Araujo VC, Demasi AP. The expresión of antioxidante enzymes in the gingival of type 2 diabtics with chronic periodontitis. Arch. Oral Biol. 2012; 57:161-168.

5. Goh SY, Cooper ME. Clinical review: the role of advanced glycation end products in progression and complications of diabetes. J. Clin. Endocrinol. Metab. 2008; 93:1143-1152.

6. Kiyoshima T, Enoki N, Kobayashi I, Nagata K, Wada H , Fujiwara $\mathrm{H}$, OokumaY, Sakai H. Oxidative stress caused by low concentration of hydrogen peroxide induces senescence- like changes in mouse gingival fibroblasts. Int. J. Mol. Med. 2012; 30:1007-1012.

7. Sugiyama S, Takehashi SS, Tokutomi FA, Yoshida A, Kobayashi F, Yoshino S, Wada-Takahashi S, ToyamaT, Watanabe K, Hamada N, Todoki K, Lee M. Gingival vascular functions are altered in type 2 diabetes mellitus modelor periodontitis model. J. Clin. Biochem. Nutr. 2012; 51:108-113.

8. Marchetti E, Monaco A, Procaccini LI, Mummolo S, Gatto R, Tete S, Baldini A, Tecco S, Marzo G. Periodontal disease: the influence of metabolic syndrome. Nutr. Metab. (London) 2012; 9: 88. 
9. Govindaraj P, Khan NA, Gopalakrishna P, Chandra RV, Vanniarajan A, Reddy AA, Sing S, Komareson R, Srinvas $G$, Thangaraj. Mitochondrial dysfunction and genetic heterogeneity in chronic periodontitis. Mitochondrion 2011; 11:504-512.

10. Esen C, Alkan BA, Kirnap M, Akgul O, ISlkoglu S, Erel O. The effect of chronic periodontits and rheumatoid arthtritis on serum and gingival crevicular fluid total antioxidant/oxidant status and oxidative stress index. J. Periodontol. 2012; 83:773-779.

11. Chapple IL, Mildward MR, Dietrich T. The prevalence of inflammatory periodontitis is negatively associated with serum antioxidants concentrations. J. Nutr. 2007b; 34:103-110.

12. Parihar A, Parihar MS, Milner S, Bhat S. Oxidative stress and antioxidative in burn injury. Burns 2008; 34:6-17.

13. DAiuto F, Nibali I, Parkar M, Patel K, Survan J, Donos N. Oxidative stress, systemic inflammation and severe periodontitits. J. Dent. Res. 2010; 89:1241-1246.

14. Bradford MM. A rapid and sensitive method for quantitation of micrograms quantities of protein utilizing the principle of protein dye binding. Anal. Biochem. 1976, 72:148-174.

15. Ellman GL. Tissue sulphydril groups. Arch. Biochem. Biophys 1959, 82: 70-77.

16 Ohkawa $\mathrm{H}$, Ohishi N, Yagi K. Assay for lipid peroxidase in animal tissues by thiobarbituric acid reaction. Anal. Biochem. 1979, 95:351-358.

17. Green LC, Wagner DA, Glogowski J, Skipper PL, Wishnok JS, Tenebaum SR. Analysis of nitrate, nitrite and [15 N] nitrate in biological fluids. Anal. Biochem. 1982, 126:131-138.

18. Meenawat A, Punn K, Srisvastava V, Menawat AS, Dolas RS, Govila V. Periodontal disease and type 1 diabetes mellitus: associations with glycemic control and complications. J. Indian. Soc. Periodontol. 2013; 17:597-600.

19. Khader YS, Damond AS, El-Qaderi SS, Alkafajei AA, Batahyha WQ. Periodontal status of diabetics compared with non diabetics: a metanalysis. J. Diab. Compl. 2006; 20: 59-68.

20. Thomas B, Ramesh A, Suresh S, Prasad BR. A comparative evaluation of antioxidant enzymes and selenium in the serum of periodontitis with diabetes mellitus type 2. Contemp. Dent. 2013; 4: $176-180$.

21 Li J,Wuliji O, LiW, Jiang ZG, Ghanbari HA. Oxidative stress and neurodegenerative disorder. Int. J. Mol. Sci. 2013; 14:2443824475.
22. Camera A, Hopps E, Caimi G. Diabetic microangiopathy: physiolpathological, clinical and therapeutics aspects. Minerva Endocrinol. 2007; 32:209-229.

23. Allen EM, Matthews JB, O'Coonor R, O'Halloran D, Chapple IL. Periodontits and type 2 diabetes: is oxidative stress the mechanistic link? Scott. Med. J. 2009; 54:41-47.

24. Canakei CF, Cicek Y, Yildirim A, Sezer U, Canakei V. Increased levels of 8-hydroxydeoxyguanosine and malondialdehyde and its relation with antioxidant enzymes in saliva of periodontitis patients. Eur. J. Dent. 2009; 3:100-106.

25. Schrader J, Rennekapmp W, Niebergali U. Cytokinee-induced osteoprotegerin expression protects pancreatic beta cells through $\mathrm{P} 38$ mitogen activated protein kinase signaling against cell death. Acta Diabetol. 2007; 50;1243-1247.

26. Engebretson S, Chertog R, Nichols A, Hey-Hadavi J, Celenti R, Grbic J. Plasma tumor necrosis factor-alfa in patients with chronic periodontitis and type 2 diabetes. J Clin. Periodontol. 2007 34:18-24.

27. Sakallioglu EE, Luftfiouglu sakalliouglu U, Diraman E, Keskiner I. Fluid dynamics of gingival in diabetic and systematically healthy periodontitis patients. Arch. Oral Biol. 2008; 53:646-651.

28. Niki E. Interaction of ascorbate and $\alpha$-tocopherol. Ann NY Acad. Sci.1987;498:186-199.

29. Iwasaki m, Manz MC, Taylor GW, Yoshihara A, Miyasaki H. Relations of serum ascorbic acid and $\alpha$-tocopherol to periodontal disease. J. Dent. Res. 2012; 91:167-172

30. Gokhale NH, Acharya AB, Patil VS, Trivedi DJ, Thakur SL. A short-term evaluation of the relationship between plasma ascorbic acid levels and periodontal disease in systematically healthy and type 2 diabetes mwllitus subjects. J. Dent. Res. 2013; 10: 93-104.

31. Meyle J, Kapitza A. Assay of ascorbico acid in gingival crevicular fluid from clinically healthy gingival sites by high performance liquid chromatography. Arch. Oral Biol. 1990; 35:319-323.

32. Suomalainen $K$, Sorsa T, Lindi O, Saari H, Kontinnen YT, Uitto VJ. Hypochlorus acid induced activation of human neutrophil and gingival crevicular fluid collagenase can be inhibited by ascorbic acid. Scand. J. Dent. Res. 1991; 99: 397-405. 